\title{
CDKN2A NM_000077.4:C.152T>A
}

National Cancer Institute

\section{Source}

National Cancer Institute. CDKN2A NM 000077.4:C.152T>A. NCI Thesaurus. Code C146929.

A nucleotide substitution at position 152 of the coding sequence of the CDKN2A gene where thymine has been mutated to adenine. 